\title{
Mechanosynthesis of a Coamorphous Formulation of Creatine with Citric Acid and Humidity-Mediated Transformation into a Cocrystal
}

\author{
Kyle B. Pekar, Jonathan B. Lefton, Christina A. McConville, Jewel Burleson, Daniel Sethio, Elfi Kraka, \\ Tomče Runčevski*
}

Department of Chemistry, Southern Methodist University, Dallas, TX 75275, United States.

truncevski@smu.edu

\begin{abstract}
Creatine is the most widely used sports supplement in the world and is frequently recognized as a dietary staple among athletes. Two major supplement formulations are currently available for purchase on the US market: creatine monohydrate and creatine hydrochloride. Creatine monohydrate has relatively low aqueous solubility, which hampers its ease of preparation by consumers. Creatine hydrochloride has excellent aqueous solubility; however, it exhibits a strong acidic taste with potentially harmful effects on dental health. New supplement formulations of creatine may boost athletic performance and, at the same time, provide better taste and convenience in preparation for consumers. Herein, we report of a mechanochemical synthesis of a 1:1 coamorphous formulation of creatine and citric acid, a-CCA. Milling of anhydrous creatine and anhydrous citric acid yielded a-CCA, which was found to be structurally stabile under dry conditions. Upon exposure to humid air, the coamorphous solid rapidly converted into a 1:1 cocrystalline formulation, c-CCA. Correspondingly, milling of creatine and citric acid, with at least one source present as a monohydrate, resulted in direct mechanosynthesis of the c-CCA cocrystal. The crystal structure of c-CCA was solved ab inito and refined from powder Xray diffraction data, and the obtained structure solution $\left(P 2_{1} / c\right.$ space group, $a=23.836(4) \AA, b=5.229(1) \AA, c=11.835(2) \AA, \alpha=$ $78.25(1)^{\circ}$ ) was evaluated by energy minimization calculations at DFT level. Close inspection of the crystal packing and hydrogenbonding network revealed the presence of creatine in zwitterionic form and of citric acid as a neutral molecule. Additionally, the coamorphous solid and the cocrystal were studied by infrared spectroscopy, differential scanning calorimetry and thermogravimetry. Aqueous solubility was evaluated using nuclear magnetic resonance spectroscopy with results demonstrating significantly improved aqueous solubility of the cocrystal $(42.1 \mathrm{~g} / \mathrm{L})$ compared to that of commercial creatine monohydrate $(13.3 \mathrm{~g} / \mathrm{L})$. The cocrystal formulation was determined to be $\sim 10 \mathrm{x}$ less acidic compared to commercial creatine hydrochloride. The simple, efficient, and scalable method of preparation, the phase-purity and high degree of crystallinity under ambient conditions and the increased solubility (compared to the creatine monohydrate) and decreased acidity (compared to creatine hydrochloride) render the 1:1 creatine:citric acid cocrystal an improved and potentially marketable creatine supplement formulation. Furthermore, the mechanosynthesis of a coamorphous formulation under dry conditions and subsequent water-mediated cocrystallization can help elucidate the catalytic role of water in cocrystal formation and can inform future crystal engineering strategies applicable to a broad range of organic, solid-state materials, particularly pharmaceutics.
\end{abstract}

\section{INTRODUCTION}

Creatine, $N$-(aminoiminomethyl)- $N$-methyl glycine (Scheme 1 ), is a naturally produced compound that functions as an intermediary for the phosphagen energy system in humans and other vertebrates. ${ }^{1}$ Numerous studies have shown that supplemented creatine is an excellent ergogenic aid reported to significantly enhance both anaerobic exercise capacity and maximal power/strength performance during sets of maximal-effort muscle contractions and single-effort sprints. ${ }^{2}$ Expectedly, its popularity as a fitness supplement soared among athletes and fostered a market currently valued at $\$ 350-550$ million in the US alone. The most frequently marketed form, creatine monohydrate, was introduced in the early 1990 s as an oral supplement consumed via dissolution in beverages. In this regard, creatine monohydrate, even with its overwhelming market share, still has a significant drawback due to its rather modest aqueous solubility $(13.3 \mathrm{~g} / \mathrm{L}) .{ }^{3}$ Creatine hydrochloride was recently introduced as an alternative supplement formulation with drastically

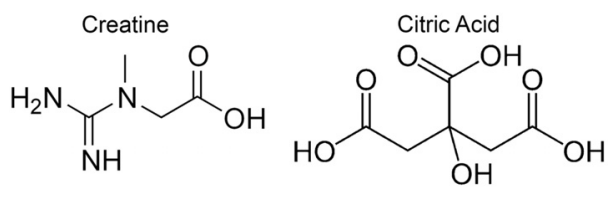

Scheme 1. Structural formulas of creatine and citric acid.

increased aqueous solubility $(\geq 150 \mathrm{~g} / \mathrm{L}) .{ }^{4}$ However, characterized by a strong acidic aftertaste, the high degree of acidity associated with hydrochloride salts can adversely impact dental health. To the best of our knowledge, creatine monohydrate and creatine hydrochloride are currently the only mass-produced formulations available within the US market. This surge in popularity of creatine, in conjunction with explosive growth of the sports supplement industry, motivates the development and 
eventual presentation of novel formulations of creatine. Several of these alleged forms have been reported without proper characterization and remain questionable in their collective legal/regulatory status. ${ }^{5}$ In the early 2000 s, creatine salts such as creatine citrate, creatine pyruvate, and creatine ascorbate were patented. ${ }^{6}$ Out of all reported formulations, only creatine monohydrate and its anhydrous forms have been thoroughly studied. In fact, creatine monohydrate represents the most extensively studied sports supplement formulations in history from a biochemical/metabolic perspective, ${ }^{1}$ in regard to supplement performance, ${ }^{2}$ and crystallographic characterization. ${ }^{7-9}$

We sought to apply crystal-engineering strategies to design a new creatine formulation with increased solubility. The same design principles used in formulating pharmaceuticals ${ }^{10-18}$ can be applied to supplements. The formation of cocrystals with biocompatible, highly soluble counterparts can be implemented to enhance the aqueous solubility of creatine, improving consumer appeal and potentially efficacy as a supplement. Similarly, the preparation of coamorphous solids has shown to be a new and potent crystal-engineering strategy for increasing the solubility and stability of pharmaceuticals. In this regard, mechanochemistry has emerged as a valuable tool for the preparation of new cocrystalline and coamorphous formulations. ${ }^{19-25}$ In its molecular structure, creatine features carboxylic and guanidino functional groups (Scheme 1), both of which are available for acid-base chemistry and cocrystal formation. We tested the propensity of creatine to engage in cocrystal formation with various cocrystal counterparts; our screening experiments indicate that creatine does not readily form cocrystals. We attribute this to the possibility of proton transfer between the carboxylic and guanidino functional groups and the formation of strong intermolecular interactions between creatine zwitterions, as observed in creatine polymorphs and hydrates. ${ }^{7-9}$ Usually, our solvothermal crystallizations resulted in precipitation of physical mixtures containing creatine and counterpart. Interestingly, we found that mechanochemical synthesis of creatine with citric acid (Scheme 1) allowed for the unique synthesis of a 1:1 formulation. We purposely chose citric acid as a counterpart as it is known to undergo glass transition upon cooling from a melt, ${ }^{26}$ furthermore this acid is proven to form cocrystals ${ }^{27,28}$ and coamorphous solids. ${ }^{29} \mathrm{We}$ hypothesize that, given its propensity to form a disordered solid, citric acid may be a good candidate for a coamorphous/glassy formulation with creatine, which could later be transformed into a cocrystal. In fact, milling of both compounds in dry conditions gave a coamorphous solid (aCCA) that is stable under dry conditions, but readily converts to a cocrystal (c-CCA) upon contact with ambient humidity. Milling in the presence of small amounts of water (via creatine hydrate, citric acid hydrate, or catalytic drop) directly yielded c-CAA. In this case, water plays a catalytic role in the crystallization c-CAA, whereas a-CCA can be considered an intermediate in the process. Herein, we report these intriguing processes and their associated solid-state structures and physicochemical properties determined by using a combination of complementary methods: high-resolution powder X-ray diffraction (PXRD), infrared (IR) spectroscopy, thermogravimetric analysis (TGA), differential scanning calorimetry (DSC), nuclear magnetic resonance (NMR) spectroscopy, and density functional theory (DFT) methods.

\section{EXPERIMENTAL SECTION}

Materials. Creatine monohydrate (purchased from GNC), citric acid anhydrate (purchased from Fisher), and citric acid monohydrate (purchased from Fisher) were used as received without further purification. Their purity was checked by NMR prior to usage. Creatine anhydrate was prepared by dehydration of creatine monohydrate at $373 \mathrm{~K}$ for $3 \mathrm{~h}$ and confirmed with TGA. The anhydrous forms of creatine and citric acid were stored in a desiccator prior use.

Mechanosynthesis of a-CCA. Creatine anhydrate $(131 \mathrm{mg}$, $1.00 \mathrm{mmol})$ and citric acid anhydrite $(192 \mathrm{mg}, 1.00 \mathrm{mmol})$ were transferred to a stainless-steel milling canister along with one stainless-steel ball bearing (4.0 g). The sample was milled for $30 \mathrm{~min}$ at $30 \mathrm{~Hz}$, resulting in a fine, chalky powder, which was transferred to a drybox under a nitrogen atmosphere.

Mechanosynthesis of c-CCA. Creatine monohydrate (149 $\mathrm{mg}, 1.00 \mathrm{mmol})$ and citric acid anhydrate $(192 \mathrm{mg}, 1.00 \mathrm{mmol})$ were transferred to a stainless-steel milling canister along with one stainless-steel ball bearing $(4.0 \mathrm{~g})$. The mixture was milled for $30 \mathrm{~min}$ at $30 \mathrm{~Hz}$, yielding a sticky solid which was removed to dry on the benchtop for $24 \mathrm{~h}$, resulting in a dry solid. Similar procedures were repeated using creatine anhydrate milled with citric acid monohydrate and creatine monohydrate milled with citric acid monohydrate, resulting in the same crystalline product as confirmed by PXRD.

PXRD. PXRD patterns were collected on a high-resolution laboratory Stoe Stadi-P powder diffractometer, operating in Debye-Scherrer (transmission) geometry. The diffractometer was equipped with a molybdenum X-ray source. Monochromatic Mo- $K \alpha_{1}$ radiation was obtained by a primary Ge(111) monochromator (centered at $0.7093 \AA$ ). Scattered X-ray intensity was simultaneously collected by two highly sensitive, linearly positioned silicon-strip (Mythen Dectris 1K) detectors. Sample preparation involved very gentle grinding of the materials in a pestle and packing in borosilicate capillaries with a $0.5 \mathrm{~mm}$ diameter. During measurements, the capillary was rotated for improved particle statistics. Diffraction data was collected at room temperature. Typically, diffraction patterns were collected in the $0-25^{\circ} 2 \theta$ range for $2 \mathrm{~h}$. The diffraction pattern used for structure solution and refinement was collected for $24 \mathrm{~h}$.

Crystal Structure Solution and Refinement. The powder diffraction pattern of c-CAA (Figure 1) was analyzed with the TOPAS-Academic V6 (Coelho Software, 2018) software. The indexing of the pattern was performed by the singular value decomposition function. ${ }^{30}$ The indexing indicated a monoclinic unit cell, with a probable $P 2{ }_{1} / c$ space group symmetry (later confirmed by a Rietveld ${ }^{31}$ refinement). Precise unit cell parameters were obtained by structureless Pawley fitting, ${ }^{32}$ using the fundamental parameter approach. ${ }^{33}$ During the Pawley fitting iterations, unit cell parameters, background coefficients (described as a Chebyshev polynomial of $10^{\text {th }}$ order), and parameters describing both the peak profile and instrumental contribution (using the simple axial model) were simultaneously varied. Once the fitting converged, the crystal structure solution process was initiated using precise unit cell parameters and a $P 2_{1} / c$ symmetry and completed by the real-space global optimization approach of simulated annealing using the Metropolis algorithm. ${ }^{34}$ To decrease the degrees of freedom, rigid bodies described in a $Z$-matrix notation were utilized. ${ }^{35}$ Based on the asymmetric unit cell volume, one creatine molecule and one citric acid molecule were used in the structure solution process. Considering the possibilities of proton transfer, as well as the 
conformational flexibility of the carboxylate group, hydrogen atoms were not included in the initial model (except for the hydrogens bound to aliphatic carbon atoms). To account for the small excess of electron density from the hydrogen atoms, the occupancy factors of the terminal oxygen atoms were set flexible within the 1-1.1 range. During the simulated annealing runs, bond distances and angles within the rigid bodies were kept fixed, whereas all possible dihedral angles were set flexible. The rigid bodies were freely translated and rotated in the asymmetric unit until convergence. Once the optimization converged to a global minimum, the model was subjected to whole-pattern Rietveld refinement. ${ }^{30}$ Hydrogen atoms were added based on geometric considerations. During the refinement, all of the aforementioned parameters were freely refined, in addition to unit cell parameters, peak shape and profile parameters, background coefficients (described as a Chebyshev polynomial), bond lengths and angles within the rigid bodies (applying soft constraints), and thermal displacement parameters (defined as a single variable for each atomic type, except for hydrogen atoms, which were calculated as the thermal displacement parameter of the bonded atom multiplied by a factor of 1.5). The Rietveld refinement converged quickly with satisfactory figures of merit and a satisfactory difference curve. The Rietveld plot is given in Figure 1, and selected parameters and figures of merit are presented in Table 1.

Table 1. Selected Structural, Crystallographic and X-ray Powder Diffraction Data for c-CAA.

\begin{tabular}{ll}
$\lambda(\AA)$ & c-CAA \\
$T(\mathrm{~K})$ & 0.7093 \\
$2 \theta$ range $\left(^{\circ}\right)$ & 293 \\
Time $(\mathrm{h})$ & $1-25$ \\
molecular formula & 24 \\
crystal system & $\mathrm{C}_{4} \mathrm{H}_{9} \mathrm{~N}_{3} \mathrm{O}_{2}: \mathrm{C}_{6} \mathrm{H}_{8} \mathrm{O}_{7}$ \\
space group & Monoclinic \\
$a(\AA)$ & $P 2{ }_{1} / c$ \\
$b\left(^{\AA}\right)$ & $23.836(4)$ \\
$c(\AA)$ & $5.229(1)$ \\
$\alpha\left(^{\circ}\right)$ & $11.835(2)$ \\
$\beta\left(^{\circ}\right)$ & 90 \\
$\gamma\left({ }^{\circ}\right)$ & $78.25(1)$ \\
$V(\AA)$ & 90 \\
$\mathrm{Z}$ & $1444.3(5)$ \\
$R_{\exp }(\%)^{\mathrm{a}}$ & 1 \\
$R_{\mathrm{p}}(\%)^{\mathrm{a}}$ & 0.02 \\
$R_{\mathrm{wp}}(\%)^{\mathrm{a}}$ & 3.25 \\
$R_{\mathrm{Bragg}}(\%)^{\mathrm{a}}$ & 4.33 \\
$\mathrm{No}$ of variables & 1.03 \\
\hline
\end{tabular}

a The figures of merit are as defined in TOPAS-Academic V6.

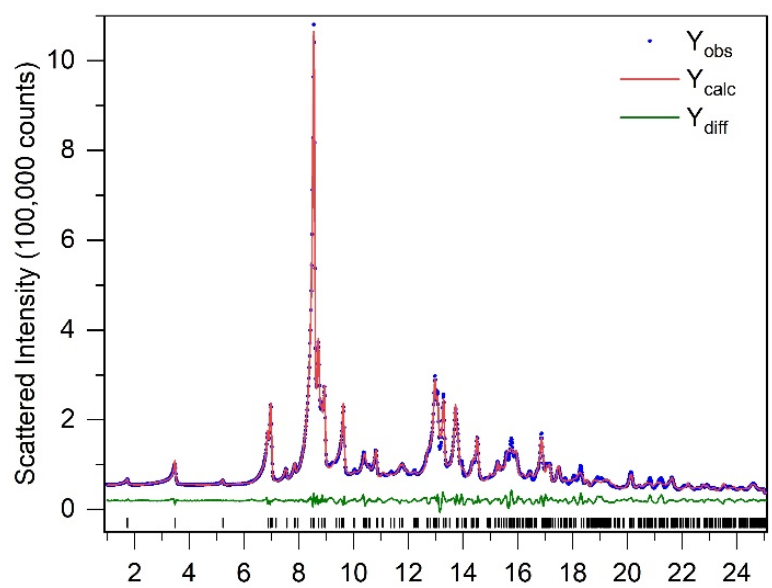

Figure 1. Powder diffraction pattern and Rietveld refinement plot for c-CAA. Measured scattered X-ray intensity is presented as blue dots, the simulated pattern is presented as a red line, the difference curve between the measured and simulated patterns is presented as a green line, and Bragg reflections are given as blue bars (X-ray $\lambda=0.7093 \AA$ ).

Theoretical Calculations. Geometry optimizations and vibrational frequency calculations for creatine, citric acid, and their cocrystal were carried out utilizing density functional theory $(\mathrm{DFT})^{36}$ with periodic boundary conditions, based on the B3LYP functional ${ }^{37,38}$ augmented with a modified empirical dispersion term (B3LYP-D*). ${ }^{39}$ The B3LYP-D* has proven to adequately account for electron correlation in crystalline systems and describing noncovalent interactions including hydrogen bonding..$^{40,41}$ The pob-TZVP Gaussian-type basis set of triple- $\zeta$ quality was employed for describing $\mathrm{C}, \mathrm{H}, \mathrm{O}$, and $\mathrm{N} .{ }^{42}$ All DFT calculations were performed with the Crystal17 program package. ${ }^{43}$ Geometry optimizations including a full relaxation of both atomic positions and lattice parameters were carried out using the Monkhorst-Pack ${ }^{44}$ grid of $10 \times 10 \times 8$ in combination with the convergence criteria of $10^{-7}$ for the Coulomb and exchange integrals. Harmonic vibrational frequencies and corresponding normal modes were calculated at the center of the Brillouin zone $(\Gamma \text { point })^{44}$ to ensure that the relaxed structures correspond to local energy minima.

IR Spectroscopy. IR spectra were collected with a Fourier transform infrared (FTIR) Thermo Fisher Nicolet iS50 spectrometer. The samples were prepared by grinding $1 \mathrm{mg}$ of the sample with $100 \mathrm{mg}$ of $\mathrm{KBr}$ and then pressing into a pellet.

TGA. The thermogravimetric analyses of creatine anhydrate, creatine monohydrate, citric acid anhydrate, a-CCA, and cCCA were performed with a Netzsch TG 209 F3 Tarsus instrument. Each sample was placed in an alumina crucible and heated from 25 to $1000{ }^{\circ} \mathrm{C}$ at a heating rate of $10{ }^{\circ} \mathrm{C} / \mathrm{min}$ under a constant flow of nitrogen.

DSC. DSC measurements for a-CCA and c-CCA were performed on a Netzsch DSC 214 Polyma instrument in hermetically sealed aluminum pans, heated from 30 to $140{ }^{\circ} \mathrm{C}$ at a rate of $10^{\circ} \mathrm{C} / \mathrm{min}$ under a constant flow of nitrogen. 
NMR Spectroscopy. All ${ }^{1} \mathrm{H}-\mathrm{NMR}$ spectra were collected on a Jeol $500 \mathrm{MHz}$ spectrometer with a variable temperature probe. Experiments were setup with a $45^{\circ}$ pulse and $10 \mathrm{~s}$ relaxation delay, with 16 scans per measurement.

Solubility Measurements. The aqueous solubility of creatine monohydrate and c-CCA was determined via NMR spectroscopy. The samples were weighed such that the maximum achievable concentration was $0.50 \mathrm{M}$. NMR tubes were prepared by first adding the creatine monohydrate $(45 \mathrm{mg})$ or cCCA $(58 \mathrm{mg})$, followed by $600 \mu \mathrm{L}$ of the internal standard solution. The NMR tube was vigorously shaken with a Vortex 2 Genie to form a suspension, which was then settled with the aid of centrifugation. NMR experiments were then measured with a relaxation delay of $10 \mathrm{~s}$, allowing for 15-min dissolution periods after raising the temperature. The dissolved content was measured at $25,35,45$, and $55^{\circ} \mathrm{C}$.

pH Measurements. Three $15 \mathrm{~mL}$ solutions of $0.067 \mathrm{M}$ creatine monohydrate (149 mg), c-CCA (341 mg), and creatine hydrochloride (168 mg) were prepared and measured at room temperature with a Mettler Toledo SevenCompact $220 \mathrm{pH} / \mathrm{Ion} \mathrm{Me}-$ ter and InLab Expert Pro-ISM probe stored in $3 \mathrm{M} \mathrm{KCl}$ solution. Quantitative dissolution/equilibration was allowed to take place overnight prior to the obtaining of $\mathrm{pH}$ values. The SevenCompact 220 Meter calibration was performed using the 2, 4, 7, and 10 predefined buffer group setting with standard solutions. Between each measurement (calibration and experimental), the probe was rinsed thoroughly with deionized water and dried with a Kimwipe.

\section{RESULTS AND DISCUSSION}

Mechanosynthesis and characterization of a-CCA. Our attempts to form new formulations of creatine with various coformers by solvatothermal methods were unsuccessful; numerous crystallizations at various conditions resulted in polycrystalline mixtures. In fact, creatine is poorly soluble in various solvents, limiting the design of a broad screening procedure.
Therefore, we sought to use mechanochemistry as an alternative strategy for cocrystal screening. One of our primary targets was producing new formulation of creatine with citric acid-a foodgrade and inexpensive chemical that can be used to alter various physicochemical properties of solid-state formulations, such as solubility and taste. Moreover, citric acid is known to undergo glass transition upon cooling from a melt, ${ }^{26}$ which is indicated towards the possibility for formation of coamorphous/glassy solids. In fact, ball milling of equimolar amounts of creatine anhydrate with citric acid anhydrate led to a supramolecular reaction and formation of a coamorphous formulation, a-CAA. The powder diffraction pattern of a-CAA, shown in Figure $2 a$, features a complete disappearance of Bragg peaks and presence of a broad halo from 4 to $20^{\circ} 2 \theta$ with X-ray scattering maximum at $8^{\circ} 2 \theta$. As a control experiment, we performed ball milling of phase-pure creatine anhydrate and phase-pure citric acid anhydrate. As shown in Figure 2a, neither of these experiments resulted in a polymorphic phase transition or amorphization. Therefore, the amorphization is not attributed to the mechanical impact, rather to a mechanochemical reaction.

To gain further insight into the local structure of the product obtained by milling, the sample was studied using IR spectroscopy. Figure $2 \mathrm{~b}$ presents the IR spectrum of the coamorphous sample, together with the IR spectra of samples of phase-pure creatine anhydrate and citric acid anhydrate, prepared under identical conditions. The spectrum of the milled sample has markedly different spectral features compared to the spectra of the pure compounds. Expectedly, the spectrum of a-CCA features a significant peak broadening and loss of fine structure, both of which are concomitants of the loss of long-order periodicity. Namely, in the coamorphous formulation, each creatine and citric acid building block has a slightly different local structure that leads to a different local environment around the vibrating atoms, hence giving rise to red/blue shifts and broadening of the corresponding IR peaks.
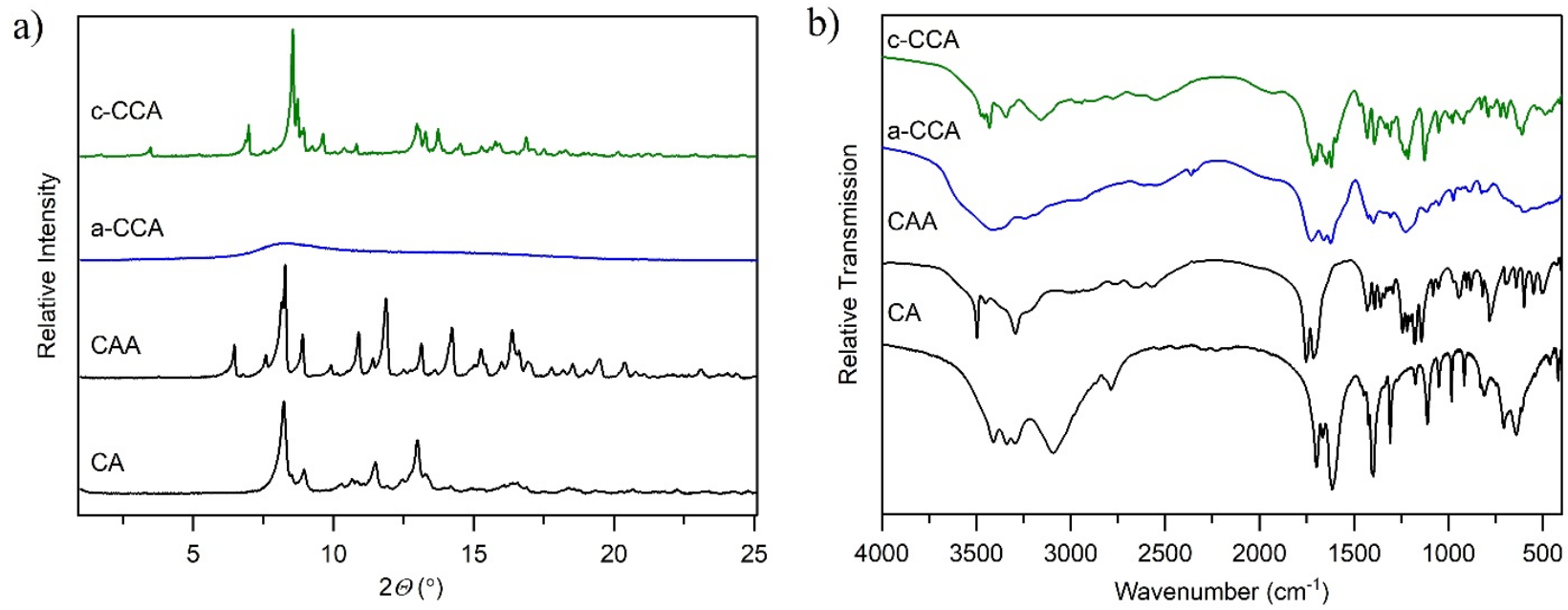

Figure 2. a) PXRD patterns and b) IR spectra of a-CCA (blue lines) and c-CCA (green lines). The PXRD patterns and IR spectra of milled samples of phase-pure creatine anhydrite and phase-pure citric acid anhydrite are shown for reference (black lines). 

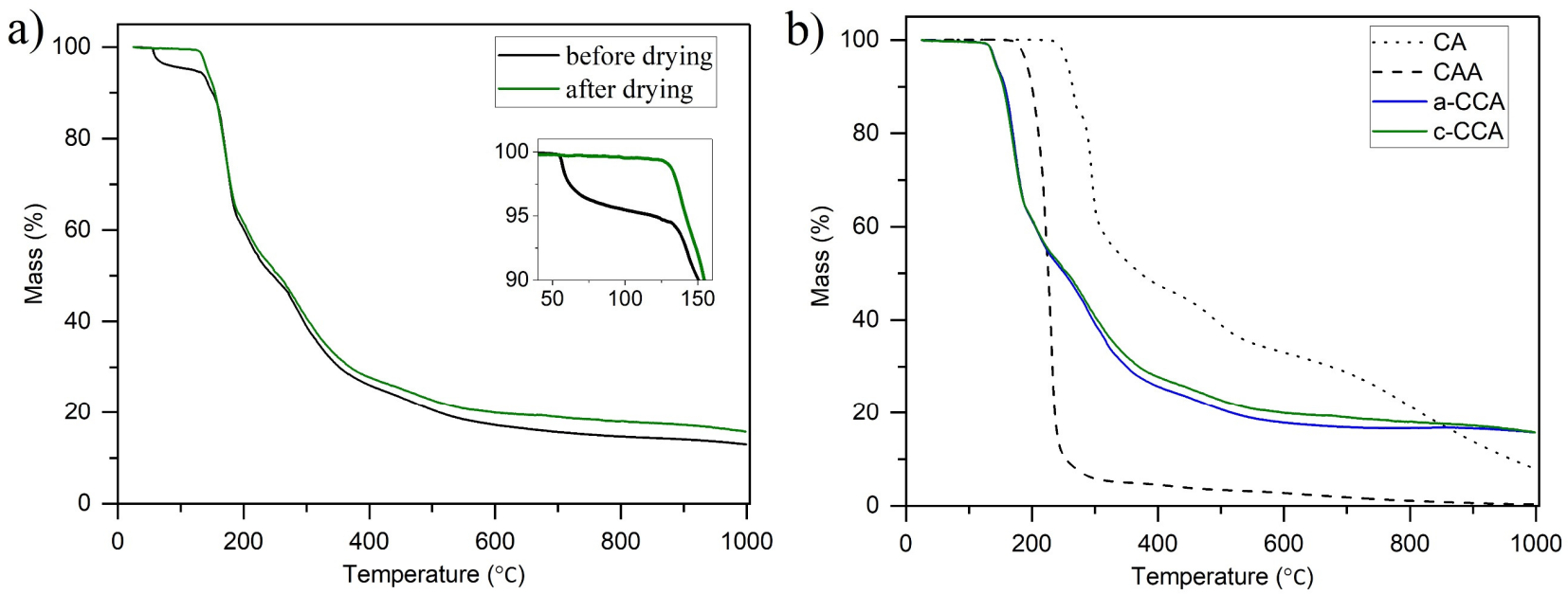

Figure 3. a) TGA curves of c-CAA measured directly after milling (black line) and after drying of the sample for $24 \mathrm{~h}$ at room temperature (green line). b) TGA curves of a-CAA (blue line) and c-CAA (green line). The TGA curves of phase-pure creatine anhydrite and phase-pure citric acid anhydrite are shown for reference (black dotted lines).

The thermal analysis of a-CAA indicated that the coamorphous material is thermally stable up to a temperature of 129 ${ }^{\circ} \mathrm{C}$, as shown in Figure 3a. The decomposition profile features two consecutive steps, which can be assigned to the decomposition of one equivalent of citric acid, followed by the decomposition of one equivalent of creatine, confirming the expected 1:1 stoichiometry. The decomposition temperature of a-CAA is significantly lower than the decomposition temperatures of phase-pure creatine anhydrate $\left(220^{\circ} \mathrm{C}\right)$ and citric acid anhydrate $\left(164^{\circ} \mathrm{C}\right)$, shown in Figure $3 \mathrm{~b}$. The different thermal decomposition pattern of a-CAA further supports the hypothesis that the milling product is a coamorphous formulation and not a physical mixture of amorphous starting materials. In case of a physical mixture, the decomposition profile would mimic a superimposed combination of the reactants' decomposition curves.

Mechanosynthesis and characterization of c-CCA. It is well-established that small, catalytic amounts of liquids can dramatically change the course of a mechanochemical reaction and lead to the formation of different products. ${ }^{21-26} \mathrm{We}$ were particularly interested in studying the effect of water on the milling reaction, considering that creatine frequently comes in the form of a monohydrate. Moreover, water molecules can be incorporated into solid formulations to form the corresponding hydrates, ${ }^{25,45}$ hence allowing for the synthesis of coamorphous of cocrystal hydrates. Additionally, water can serve as an agent for liquid assisted grinding (LAG), ${ }^{23}$ leading to products different than those made by dry milling. We tested different strategies for introducing water molecules into the mechanochemical process. First, creatine anhydrate and citric acid anhydrate were milled together with $0.056 \mu \mathrm{L}$ of water per mg of sample (typical for LAG). Second, water was introduced in a crystalline form, as a compositional part of either creatine monohydrate, citric acid monohydrate, or both. Regardless of the origin of the water molecules, the mechanochemical reactions consistently resulted in a highly crystalline product. The powder diffraction pattern of the milling product is presented in Figure 2a. Close inspection of the pattern revealed that the material exists as a single phase, without detectable amounts of crystalline phases of creatine and/or citric acid. Thorough spectroscopic and thermal analyses indicated that the crystalline product is an anhydrous cocrystal of creatine and citric acid, c-CAA, with a 1:1 stoichiometry. The IR spectrum of c-CAA is remarkably similar to the IR spectrum of a-CAA, shown in Figure 2b. The similarity between the IR spectra of both formulations points to similarities in their local structures and is indicative of a $1: 1$ stoichiometry. The IR bands in the spectrum of c-CAA are expectedly sharper considering the high crystallinity. The 1:1 stoichiometry was confirmed by thermal analyses; the TGA profile of c-CAA features a small mass loss $(\sim 4.8 \%)$ at low temperature $\left(57^{\circ} \mathrm{C}\right)$, which is characteristic for a loss of physisorbed water molecules, followed by a two-step decomposition profile. The similar TGA profiles of dried c-CAA and a-CAA, shown in Figure 3, further confirm that the coamorphous and the cocrystalline formulation have the same stoichiometry and similar local structures. It should be mentioned that physisorbed water amounts are present only after synthesis of the cocrystal. Drying of the material on the benchtop for over $24 \mathrm{~h}$ results in a complete removal of the water and production of anhydrous solid, as shown in the TGA curves presented Figure $3 \mathrm{a}$.

Crystal Structure Description of c-CAA. The structure and stoichiometry of c-CAA were proven by detailed structural analyses. The high crystallinity and phase purity of the material allowed for $a b$ initio crystal structure solution and Rietveld refinement from the PXRD data. The Rietveld refinement revealed that the c-CCA cocrystal adopts an ordered structure that can be described in the monoclinic $P 2_{1} / c$ space group. The asymmetric unit consists of one creatine and one citric acid counterpart. The crystal packing, shown in Figure 4, features creatine and citric acid double layers, repeating in the direction of the $a$-crystallographic axis. In the creatine double layer, each creatine interacts with three symmetrically equivalent creatine molecules, with hydrogen bonding with the carboxylic and 

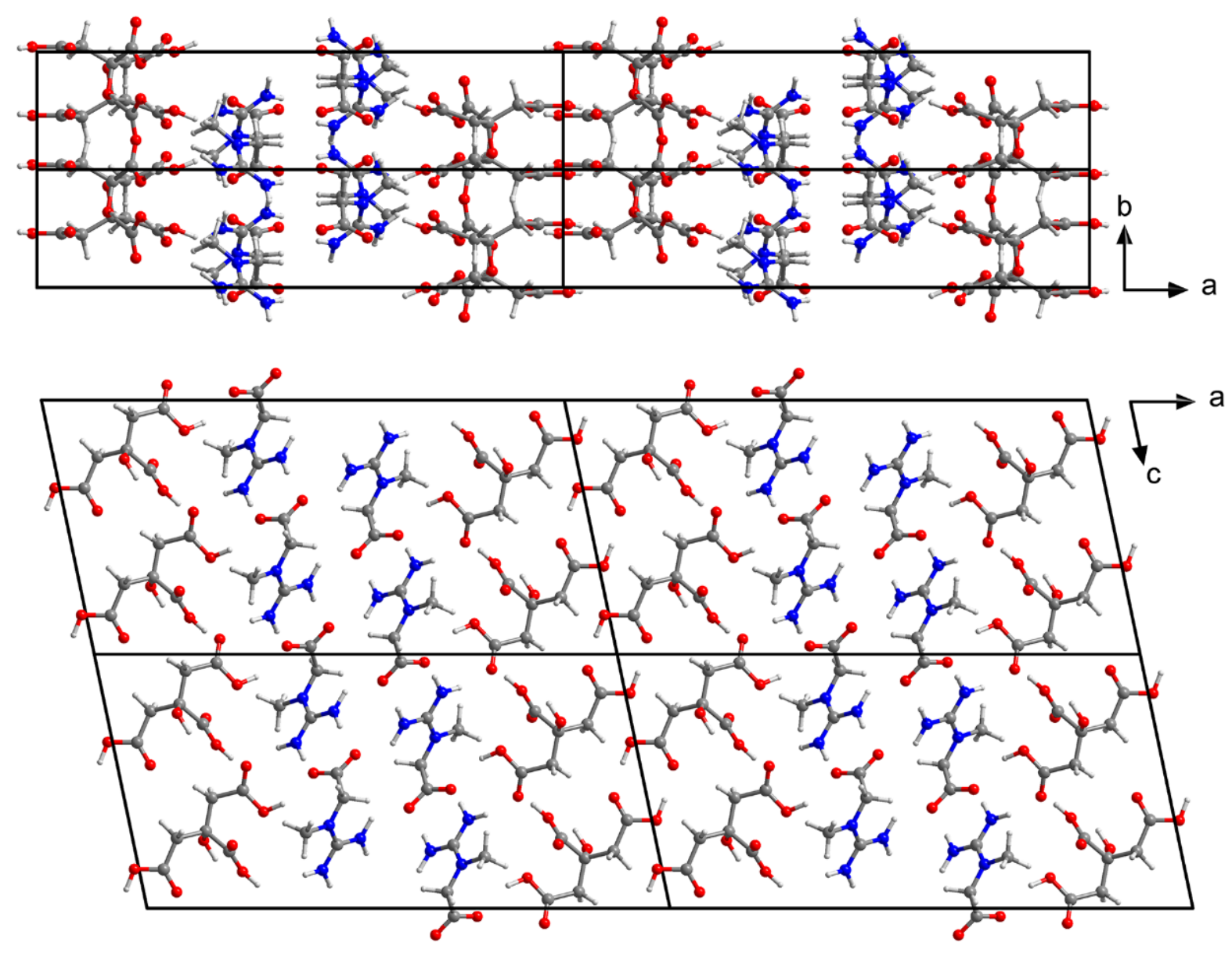

Figure 4. Crystal-packing diagram of c-CCA presented along the $c$-crystallographic axis (upper panel) and the $b$-crystallographic axis (upper panel). The packing is presented with four unit cells. Hydrogen, carbon, oxygen and nitrogen atoms are represented with white, gray, red and blue, respectively.

guanidino functional groups, establishing two $R_{2}^{2}(8)$ and one $R_{2}^{2}(14)$ supramolecular graph sets, ${ }^{46}$ in addition to various intermolecular chains, $C_{2}^{2}$, and rings $R_{6}^{6}$. The close intermolecular oxygen $\cdots$ nitrogen contacts $(2.73(5) \AA, 2.72(8) \AA$ and $2.90(5)$ $\AA$, Figure 4) indicate strong hydrogen bonds between symmetrically equivalent molecules. Similarly, in the citric acid double layer, each citric acid molecule interacts with five symmetrically equivalent molecules, with the carboxylic functional groups forming one typical $R_{2}^{2}(8)$ synthon (with a close oxygen $\cdots$ oxygen contact of $2.66(8) \AA$ ) and several intermolecular chain as well as $R_{6}^{6}$ intermolecular rings. The creatine and citric acid double layers are connected by two hydrogen bonds, one between the carboxylic groups of creatine and citric acid (oxygen $\cdots$ oxygen, 2.9(2) $\AA$ ) and another, weaker one between the guanidino group and the carboxylic group (nitrogen $\cdots$ oxygen, 3.1(7) $\AA$ ). The hydrogen bonding scheme of creatine and citric acid is dominated by discrete, finite hydrogen-bonding patterns, forming various discrete graph sets, in addition to a $C_{2}^{2}$ intermolecular chain.
To inspect the stability of the experimental crystal structure, we performed detailed DFT energy calculations, using the B3LYP-D* functional. As a control experiment, we performed identical geometry optimizations on the reported crystal structures of creatine monohydrate and citric acid. The geometry optimizations of each structure confirmed that the experimental models are energetically stable. Small deviations are observed in regard to the unit cell parameters; these can be assigned to the presence of strain and stress in the crystal structures, which are not accounted for by the theoretical calculations. Importantly, the overall crystal packing, and particularly the hydrogen-bonding network, are found to be in excellent agreement between experiment and theory. The optimized crystal structure is provided as a CIF in the SI.

Accurate classification of a solid-state formulation as a salt or as a cocrystal can impact the interpretation of the physicochemical properties ${ }^{47}$ and inform future applications of crystal engineering strategies as well as patenting procedures ${ }^{48}$ Creatine and citric acid can form both a cocrystal and a salt. Due to the intrinsic limitations of the method, laboratory PXRD cannot 


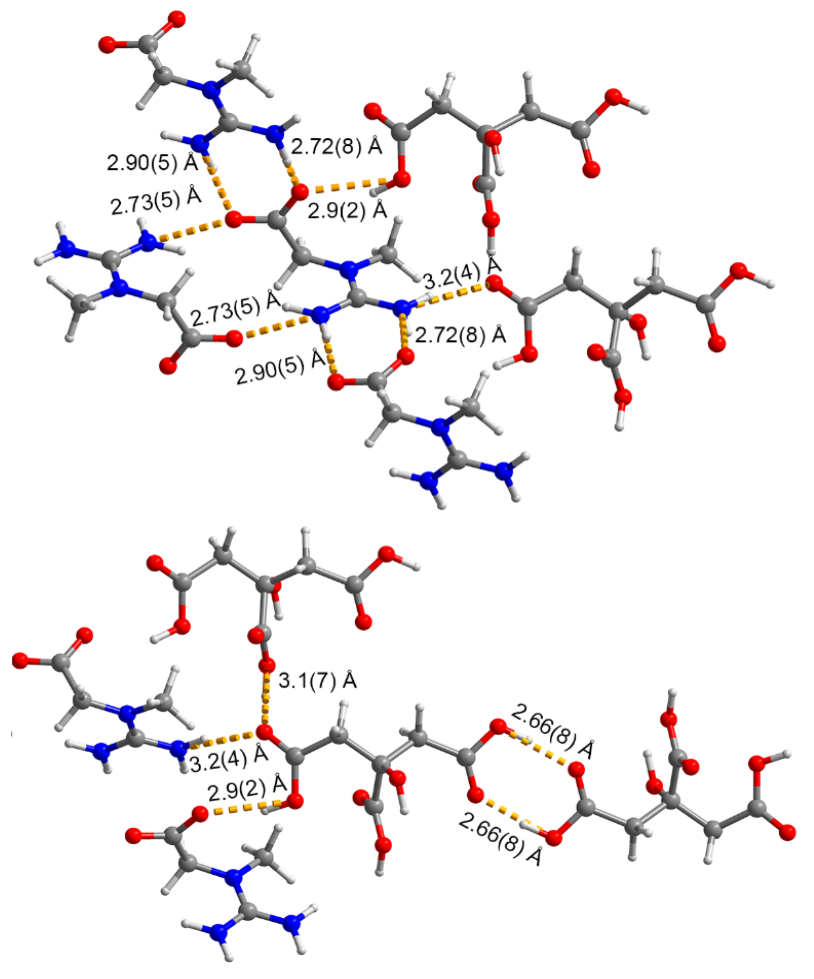

Figure 5. Selected hydrogen-bonding pattern around the creatine and citric acid in the structure of c-CCA. Hydrogen bonds are presented with dotted yellow bonds. Hydrogen, carbon, oxygen and nitrogen atoms are shown in white, gray, red and blue, respectively.

be used to detail the position of the hydrogen atoms in the structure; however, salt/cocrystal characterization can be successfully performed by considering the supramolecular synthons and the $\mathrm{p} K_{\mathrm{a}}$ rule. ${ }^{49}$ Citric acid has $\mathrm{p} K_{\mathrm{a} 1}=3.13, \mathrm{p} K_{\mathrm{a} 2}=4.76$ and $\mathrm{p} K_{\mathrm{a} 3}=6.39$, whereas creatine has $\mathrm{p} K_{\mathrm{a} 1}=3.5$ and $\mathrm{p} K_{\mathrm{a} 2}=12.46$. An interaction between the guanidino group of creatine and a carboxylic group of citric acid $\left(\Delta \mathrm{p} K_{\mathrm{a}}=9.33\right)$ would result in the formation of salt, whereas the interaction between the carboxylic groups would result in a cocrystal. Inspection of the hydrogen-bonding pattern in the structure of c-CAA clearly outlines a $R_{2}^{2}(8)$ synthon between symmetrically equivalent creatine molecules, with $\Delta \mathrm{p} K_{\mathrm{a}}=8.96$ and relatively close oxygen $\cdots$ nitrogen distances of 2.72(8) $\AA$ and 2.90(5) $\AA$ (Figure 5). It is safe to assume that the carboxylic groups of creatine can protonate neighboring guanidino groups and form creatine zwitterions. At the same time, the interaction between the guanidino group of creatine and the carboxylic group of citric acid is comparatively weaker, as evidenced by a significantly longer nitrogen $\cdots$ oxygen distance of 3.1(7) $\AA$. Furthermore, the presence of a $R_{2}^{2}(8)$ synthon between two creatine molecules excludes the possibility of a simultaneous presence of a protonated carboxylic and protonated guanidino groups.
Coamorphous-to-Cocrystal Transformations. An intrinsic characteristic of amorphous solids is their lower stability compared to their thermodynamically most stable crystalline counterparts. Therefore, we sought to investigate the stability of the a-CAA as a function of temperature and humidity.

Figure 6 presents the DSC curves of a-CAA and c-CAA collected from 30 to $140{ }^{\circ} \mathrm{C}$. c-CAA is characterized by a melting endothermic peak centered at $126^{\circ} \mathrm{C}$ and a thermal decomposition peak centered at $138{ }^{\circ} \mathrm{C}$, complementary to the TGA results. Heating of a-CAA leads to two distinct thermal events. First, an exothermic event is observed at $102{ }^{\circ} \mathrm{C}$ associated with crystallization of the coamorphous phase. Second, the crystalline phase undergoes melting at $135^{\circ} \mathrm{C}$, followed by thermal decomposition. Considering the similar thermal decomposition profiles of a-CAA and c-CAA (Figure 3a), we hypothesize that heating of a-CAA leads to crystallization into a cocrystal with a crystal structure closely related to c-CAA. Variable-temperature PXRD experiments, as well as quenching of the crystalline phase to room temperature, are underway.

Next, we tested the behavior of C-CAA and a-CAA exposed to air humidity. The effects of humidity on solid formulations are well-documented in a number of processes, including hydration of anhydrites, ${ }^{50}$ polymorphic,${ }^{51}$ and amorphous-to-crystalline transformations, ${ }^{52}$ and cocrystal syntheses. ${ }^{53}$ Water plays a crucial role in the synthesis of c-CAA, and the cocrystal exhibits hygroscopicity, physisorbing water molecules during the milling that can be later removed by aging at room temperature overnight in air (Figure 3b). On the contrary, a-CAA can be prepared only under dry experimental conditions. To test the effect of humidity on a-CAA, we exposed a freshly prepared coamorphous sample to ambient humidity for $24 \mathrm{~h}$. As a control experiment, we stored the same amount of sample for $24 \mathrm{~h}$ in a drybox. Figure 7 presents the PXRD patterns of the sample before and after aging at ambient and dry conditions. Stored in a drybox, a-CAA remained amorphous, as evidenced by the diffraction pattern. On the contrary, exposure to ambient humidity led to crystallization of the sample; observed by the sample first

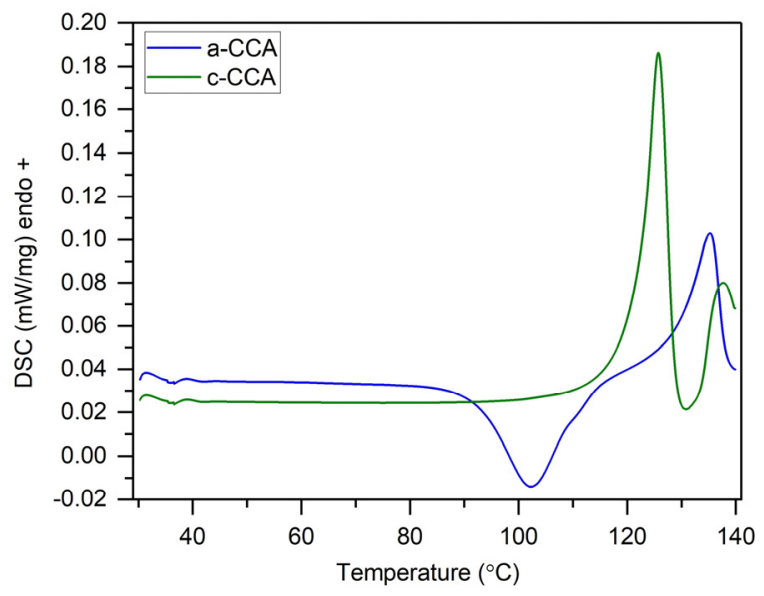

Figure 6. DSC curves of a-CCA (blue curve) and c-CCA (green curve), showing melting of c-CCA at $126^{\circ} \mathrm{C}$, crystallization of a-CCA at $102{ }^{\circ} \mathrm{C}$, and melting at $135^{\circ} \mathrm{C}$. 


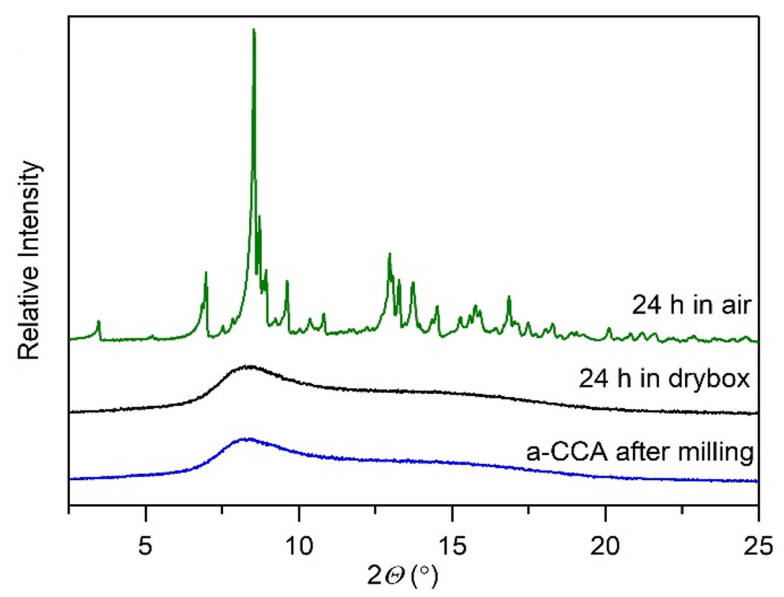

Figure 7. PXRD patterns of a-CCA collected immediately after mechanosynthesis (blue curve), after 24-hr drybox storage (black curve), 24-hr storage under ambient conditions (green curve, transformed into c-CCA).

becoming sticky and then yielding a dry crystalline solid after being dried overnight. Rietveld refinement confirmed that the crystallized sample has the same crystal structure as c-CAA. In our experiments, we did not observe deliquescence of a-CAA or C-CAA.

We hypothesize that the humidity-mediated crystallization of a-CAA into c-CAA proceeds in two steps. First, owing to its hygroscopic nature, a-CAA adsorbs water from the air. Next, the regions of a-CAA which are in contact with water molecules exhibit higher molecular mobility, which can be associated with the plasticizing effects of water. ${ }^{54}$ The enhanced molecular mobility of creatine and citric acid in the amorphous state allows for local reorganization into the thermodynamically stable local geometry. The well-defined, directional hydrogenbonding network facilitates an attainment of long-order periodicity and crystallization. Similar effects of moisture-enhanced and amorphous-mediated cocrystallization have been observed for pharmaceutical cocrystals. ${ }^{55,56}$

Solubility. The aqueous solubility of creatine in c-CAA at room temperature, determined using NMR spectroscopy, is $42.1 \mathrm{~g} / \mathrm{L}$. In comparison, the aqueous solubility determined for creatine anhydrate is $15.3 \mathrm{~g} / \mathrm{L}$. Figure 8 presents a plot of the molarity of the c-CAA and creatine monohydrate as a function of temperature. Cocrystallization of creatine with citric acid led to a nearly three-fold improvement of aqueous solubility compared to the marketed creatine monohydrate $(13.3 \mathrm{~g} / \mathrm{L}){ }^{3}$

Acidity. Considering the strong acidic aftertaste of creatine hydrochloric, decreasing the acidity of the formulations is highly sought after. Our measurements of the $\mathrm{pH}$ of c-CCA solution resulted in a $\mathrm{pH}$ value of 3.32, whereas the $\mathrm{pH}$ of creatine hydrochloride solution was found to be 2.41 . The ten-fold decrease of acidity of c-CCA may alleviate the adverse effects on dental health and improve the palatability.

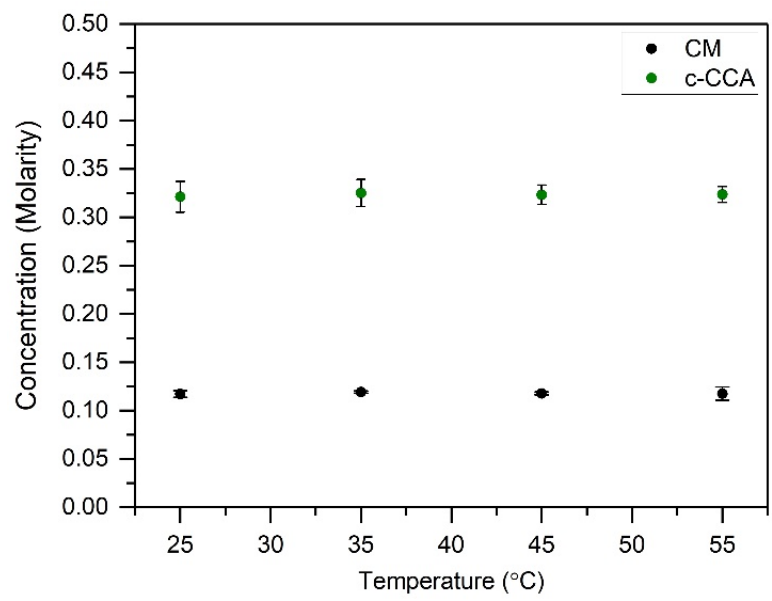

Figure 8. Aqueous solubility of c-CAA and marketed creatine monohydrate presented as the change in molarity as a function of temperature.

\section{CONCLUSIONS}

In summary, we present a mechanochemical route for the preparation of coamorphous (a-CAA) and cocrystal (c-CAA) formulations of creatine with citric acid. Mechanochemical treatment of equimolar amounts of anhydrous reactants in dry conditions leads to the formation of a-CAA, whereas milling in the presence of humidity provides the formation of c-CAA. Water can be introduced in the system as a LAG agent or as a crystalline part of the starting materials (either as creatine monohydrate, citric acid monohydrate or a combination of both).

The high crystallinity of the cocrystal allowed for $a b$ initio crystal structure solution and Rietveld refinement using the collected powder diffraction data. It was found that the asymmetric unit of the crystal structure consists of one creatine and one citric acid counterparts. Close inspection of the intramolecular synthons and the hydrogen donor-acceptor distances between the guanidino and carboxylic groups of neighboring creatine molecules indicated to the occurrence of a proton transfer and formation of creatine zwitterions. Citric acid exists in a neutral form, engaging with neighboring citric acid molecules and creatine zwitterions within a well-defined hydrogen-bonded network. The crystal structure solution was further validated by geometry optimization and energy minimization using DFT methods. The experimental and optimized structure of c-CAA were found to be in excellent agreement. To gain further insight into the local structures, a-CAA and c-CAA were studied by vibrational spectroscopy. The IR spectra of the coamorphous and cocrystal show striking similarities, and we therefore conclude that a-CAA adopts a local structure similar to the cocrystal. This conclusion is corroborated by the similar thermal decomposition profiles of both formulations, as observed by TGA. The thermal analyses confirmed that both formulations are anhydrates, consisting of 1:1 molar ratio of creatine and citric acid. 
It was found that a-CAA, while stable at dry conditions, readily transforms to c-CAA upon contact with humidity. Metastable amorphous phases have been observed as intermediates in the synthesis of various pharmaceutical cocrystals; moisturemediated cocrystal synthesis is also well-documented. In this regard, a-CAA can be considered an intermediate phase in the synthesis of C-CAA. Therefore, the isolation and thorough characterization of a-CAA may help better understand the mechanism of cocrystal formation.

Finally, the aqueous solubility of c-CAA was tested by NMR spectroscopy and compared with the solubility of marketed creatine monohydrate. It was found that cocrystallization with citric acid leads to a solubility three-fold higher than that of commercial creatine monohydrate. Additionally, the new formulation has ten-fold decrease of the acidity compared to commercial creatine hydrochloride, which can be potentially used for the improvement of the taste and alleviate the negative impact on dental health. We, therefore, conclude that c-CAA and similar cocrystals have the potential to be marketed as ergogenic aid supplements in the fitness and wellness industry.

\section{ASSOCIATED CONTENT}

\section{Supporting Information}

CIF of the c-CAA. CIF of the optimized geometry of c-CAA.

\section{Accession Codes}

CCDC XXXXX contains the supplementary crystallographic data for this paper. These data can be obtained free of charge by emailing data request@ccdc.cam.ac.uk, or by contacting The Cambridge Crystallographic Data Centre, 12 Union Road, Cambridge CB2 1EZ, UK; fax: +44 1223336033

\section{AUTHOR INFORMATION}

\section{Corresponding Author}

truncevski@smu.edu

orcid.org/0000-0003-0886-8464

\section{ACKNOWLEDGMENT}

The authors thank Southern Methodist University for providing computational resources.

\section{REFERENCES}

(1) Wyss, M; Kaddurah-Daouk R. Creatine and Creatinine Metabolism. Physiological Rev. 2000, 80, 1107-1213.

(2) Kreider, R. B. Effects of creatine supplementation on performance and training adaptations. Mol. Cell. Biochem. 2003, 244, 89-94.

(3) The Merck Index Online. 2020. [online] Available at: $<$ https://www.rsc.org/merck-index $>$ (Accessed 16 September 2020).

(4) Miller, D.W.; Vennerstrom, J.L.; Faulkner, M.C. Creatine oral supplementation using creatine hydrochloride salt. U.S. Patent No. 7,608,641 B2, Oct. 27, 2009.

(5) Jaeger, R.; Purpura, M.; Shao, A.; Inoue, T.; Kreider, R. B. Analysis of the efficacy, safety, and regulatory status of novel forms of creatine. Amino Acids 2011, 40, 1369-1383.

(6) Jager, R.; Purpura, M.; Ortenburger, G. CREATINE/CITRIC ACID COMPOUND, METHOD FOR THE PRODUCTION OF THE SAME AND THE USE THEREOF. US 2004/0077719 A1.

(7) Mendel, H; Hodgkin D. C. The crystal structure of creatine monohydrate. Acta Crystallogr. 1954, 7, 443-446.

(8) Arlin, J.-B.; Bhardwaj, R. M.; Johnston, A.; Miller, G. J.; Bardin, J.;MacDougall, F.; Fernandes, P.; Shankland, K.; David, W. I.
F.;Florence, A. J. Structure and stability of two polymorphs of creatine and its monohydrate. Cryst. Eng. Comm. 2014, 16, 8197-8204.

(9) Braun, D. E.; Orlova, M.; Griesser, U. J. Creatine: Polymorphs Predicted and Found. Cryst. Growth Des. 2014, 14, 895-4900.

(10) Schultheiss, N.; Newman, A. Pharmaceutical Cocrystals and Their Physicochemical Properties. Cryst. Growth Des. 2009, 9, 2950-2967.

(11) Good, D. J.; Rodríguez-Hornedo. N. Solubility Advantage of Pharmaceutical Cocrystals. Cryst. Growth Des. 2009, 9, 2252 2264.

(12) Duggirala, N. K.; Perry, M. L.; Zaworotko, M. J. Pharmaceutical cocrystals: along the path to improved medicines. Chem. Commun. 2016, 52, 640-655.

(13) Bolla, G.; Nangia, A. Pharmaceutical cocrystals: walking the talk. Chem. Commun. 2016, 52, 8342-8360.

(14) Sander, J. R. G.; Bučar, D.-G.; Henry, R. F.; Zhang, G. G. Z.; MacGillivray, L. R. Pharmaceutical nano-cocrystals: sonochemical synthesis by solvent selection and use of a surfactant. Angew. Chem. Int. Ed. 2010, 49, 7284-7288.

(15) Bethune, S. J.; Huang, N.; Jayasankar, A.; Rodríguez-Hornedo, N. Understanding and Predicting the Effect of Cocrystal Components and $\mathrm{pH}$ on Cocrystal Solubility. Cryst. Growth Des. 2009, 9, 3976-3988

(16) Babu, N. J.; Nangia, A. Solubility Advantage of Amorphous Drugs and Pharmaceutical Cocrystals. Cryst. Growth Des. 2011, 11, 2662-2679.

(17) Alonzo, D. E.; Zhang, G. G. Z.; Zhou, D.; Gao, Y.; Taylor, L. S Understanding the behavior of amorphous pharmaceutical systems during dissolution. Mol. Pharmaceutics 2010, 27, 608-618.

(18) Dengale, S. J.; Grohganz, H.; Rades. T.; Löbmann, K. Recent advances in co-amorphous drug formulations. Ad. Drug Delivery Rev. 2016, 100, 116-125.

(19) Braga, D.; Mainia, L.; Grepioni, F. Mechanochemical preparation of co-crystals. Chem. Soc. Rev. 2013, 42, 7638-7648.

(20) Friščić, T. Supramolecular concepts and new techniques in mechanochemistry: cocrystals, cages, rotaxanes, open metal-organic frameworks. Chem. Soc. Rev. 2012, 41, 3493-3510.

(21) Hasa, D.; Rauber, G. S.; Voinovich, D.; Jones, W. Cocrystal Formation through Mechanochemistry: from Neat and Liquid-Assisted Grinding to Polymer-Assisted Grinding. Angew. Chem. Int Ed. 2015, 127, 7479-4783.

(22) Weyna, D. R.; Shattock, T.; Vishweshwar, P.; Zaworotko, M. J. Synthesis and Structural Characterization of Cocrystals and Pharmaceutical Cocrystals: Mechanochemistry vs Slow Evaporation from Solution. Cryst. Growth Des. 2009, 9, 1106-1123.

(23) Karki, S.; Friščić, T.; Jones, W.; Motherwell, W. D. S. Screening for Pharmaceutical Cocrystal Hydrates via Neat and Liquid-Assisted Grinding. Mol. Pharmaceutics 2007, 4, 347-354.

(24) Halasz, I.; Puškarić, A.; Kimber, S. A. J.; Beldon, P. J.; Belenguer, A. M.; Adams, F.; Honkimäki, V.; Dinnebier, R. E.; Patel, B.; Jones, W.; Štrukil, V.; Friščić, T. Real-Time In Situ Powder Xray Diffraction Monitoring of Mechanochemical Synthesis of Pharmaceutical Cocrystals. Angew. Chem. Int. Ed. 2013, 125, 11752-11755.

(25) Friščić, T.; Mottillo, C.; Titi, H. M. Mechanochemistry for Synthesis. Angew. Chem. Int. Ed. 2020, 59, 1018-1029.

(26) Lu, Q.; Zografi, G. Properties of Citric Acid at the Glass Transition. J. Pharm. Sci. 1997, 86, 1374-1378.

(27) Lu, Q.; Dun, J.; Chen, J-M.; Liu, S.; Sun, C. C. Improving solidstate properties of berberine chloride through forming a salt cocrystal with citric acid. Int. J. Pharma. 2019, 554, 14-20.

(28) Deng, J.-H.; Lu, T.-B, Sun, C. C.; Chen, J.-M. Dapagliflozin-citric acid cocrystal showing better solid state properties than dapagliflozin. Eur. J. Pharma. Sci. 2017, 104, 255-261.

(29) Wang. J.; Chang, R.; Zhao, Y.; Zhang, J.; Zhang, T.; Fu, Q.; Chang, C.; Zeng, A. Coamorphous Loratadine-Citric Acid System with Enhanced Physical Stability and Bioavailability. AAPS Pharm. Sci. Tech. 2017, 18, 2541-2550. 
(30) Coelho, A. A. Indexing of powder diffraction patterns by iterative use of singular value decomposition. J. Appl. Crystallogr. 2003 , $36,86-95$.

(31) Rietveld, H. M. A profile refinement method for nuclear and magnetic structures. J. Appl. Crystallogr. 1969, 2, 65-71.

(32) Pawley, G. S. Unit-cell refinement from powder diffraction scans. J. Appl. Crystallogr. 1981, 14, 357-361.

(33) Cheary, R. W.; Coelho, A. A.; Cline, J. P. Fundamental parameters line profile fitting in laboratory diffractometers. J. Res. Natl. Inst. Stand. Technol. 2004, 109, 1-25.

(34) Andreev, Y. G.; MacGlashan, G. S.; Bruce, P. G. Ab initio solution of a complex crystal structure from powder-diffraction data using simulated-annealing method and a high degree of molecular flexibility. Phys. Rev. B: Condens. Matter Mater. Phys. 1997, 55, 12011-12017.

(35) Dinnebier, R. E. Rigid bodies in powder diffraction. A practical guide. Powder Diffr. 1999, 14, 84-92.

(36) Kohn, W.; Sham, L. J. Self-Consistent Equations Including Exchange and Correlation Effects. Phys. Rev. 1965, 140, A1133A1138.

(37) Becke, A. D. Density-Functional Exchange-Energy Approximation with Correct Asymptotic Behavior. Phys. Rev. A 1988, 38, 3098-3100.

(38) Lee, C.; Yang, W.; Parr, R. G. Development of the Colle-Salvetti Correlation-Energy Formula into a Functional of the Electron Density. Phys. Rev. B 1988, 37, 785-789.

(39) Civalleri, B.; Zicovich-Wilson, C. M.; Valenzano, L.; Ugliengo, P. B3LYP Augmented with an Empirical Dispersion Term (B3LYP-D*) as Applied to Molecular Crystals. Cryst. Eng. Commun. 2008, 10, 405-410.

(40) Surov, A. O.; Voronin, A. P.; Vener, M. V.; Churakov, A. V.; Perlovich, G. L. Specific Features of Supramolecular Organisation and Hydrogen Bonding in Proline Cocrystals: A Case Study of Fenamates and Diclofenac. Cryst. Eng. Commun. 2018, 20, 6970-6981.

(41) Erba, A.; Maul, J.; Civalleri, B. Thermal Properties of Molecular Crystals Through Dispersion-Corrected Quasi-Harmonic Ab Initio Calculations: The Case of Urea. Chem. Commun. 2016, 52, 1820-1823.

(42) Peintinger, M. F.; Oliveira, D. V.; Bredow, T. Consistent Gaussian Basis Sets of Triple-Zeta Valence with Polarization Quality for Solid-State Calculations. J. Comput. Chem. 2012, 34, 451459.
(43) Dovesi, R.; Erba, A.; Orlando, R.; Zicovich-Wilson, C. M.; Civalleri, B.; Maschio, L.; Rerat, M.; Casassa, S.; Baima, J.; Salustro, S.; Kirtman, B. Quantum-Mechanical Condensed Matter Simulations with CRYSTAL. WIREs Comput. Mol. Sci. 2018, 8, e1360.

(44) Monkhorst, H. J.; Pack, J. D. Special Points for Brillouin-zone Integrations. Phys. Rev. B 1976, 13, 5188-5192.

(45) Runčevski, T.; Petruševski, G.; Makreski, P.; Ugarkovic, S.; Dinnebier, R. E. On the hydrates of codeine phosphate: the remarkable influence of hydrogen bonding on the crystal size. Chem. Commun. 2014, 50, 6970-6972.

(46) Etter, M. C. Encoding and decoding hydrogen-bond patterns of organic compounds. Acc. Chem. Res. 1990, 23, 120-126.

(47) Aakeröy, C. B.; Fasulo, M. E.; Desper, J. Cocrystal or Salt: Does It Really Matter? Mol. Pharmaceutics 2007, 4, 317-322.

(48) Trask, A. V. An Overview of Pharmaceutical Cocrystals as Intellectual Property. Mol. Pharmaceutics 2007, 4, 301-309.

(49) Cruz-Cabeza, A. J. Acid-base crystalline complexes and the pKa rule. Cryst. Eng. Commun. 2012, 14, 6362-6365.

(50) Pirttimaki, J.; Laine, E. The Transformation of Anhydrate and Hydrate Forms of Caffeine at 100-Percent RH and 0-Percent RH. Eur. J. Pharm. Sci. 1994, 1, 203-208.

(51) Yoshinari, Y.; Forbes, R. T.; York, P.; Kawashima, Y. Moisture Induced Polymorphic Transition of Mannitol and Its Morphological Transformation. Int. J. Pharm. 2002, 247, 69-77.

(52) Andronis, V.; Yoshioka, M.; Zografi, G. Effects of Sorbed Water on the Crystallization of Indomethacinfrom the Amorphous State. J. Pharm. Sci. 1997, 86, 346-351.

(53) Jayasankar, A.; Good, D. J.; Rodríguez-Hornedo, N. Mechanisms by Which Moisture Generates Cocrystals. 2007, 4, 360-372.

(54) Hancock, B. C.; Zografi, G. The Relationship between the GlassTransition Temperature and the Water-Content of Amorphous Pharmaceutical Solids. Pharm. Res. 1994, 11, 471-477.

(55) Jayasankar, A.; Somwangthanaroj, A.; Shao, Z. J.; RodríguezHornedo, N. Cocrystal Formation During Cogrinding and Storage Is Mediated by Amorphous Phase. Pharm. Res. 2006, 23, 23812392.

(56) Seefeldt, K.; Miller, J.; Alvarez-Núñez, F.; Rodríguez-Hornedo, N. Crystallization Pathways and Kinetics of Carbamazepine-Nicotinamide Cocrystals from the Amorphous State by in-Situ Thermomicroscopy, Spectroscopy and Calorimetry Studies. J. Pharm. Sci. 2007, 96, 1147-1158. 